\title{
Distribution and Source Identification of Pu in River Basins in Southern China
}

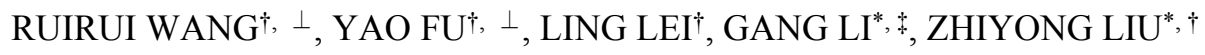

$\dagger$ State Key Laboratory of Radiation Medicine and Protection, School for Radiological and Interdisciplinary Sciences

(RAD-X) and Collaborative Innovation Centre of Radiation Medicine of Jiangsu Higher Education Institutions, Soochow University, Suzhou 215123, China.

† Key Laboratory of Ocean and Marginal Sea Geology, South China Sea Institute of Oceanology, Chinese Academy of Sciences, Guangzhou 510300, China.

*Corresponding authors. Email: gangli@scsio.ac.cn (GANG LI); liuzy@suda.edu.cn (ZHIYONG LIU); Tel: 0086-512-65883945; Fax: 0086-512-65880035.

$\perp$ These two authors contributed equally

Number of pages in Supporting Information Section: 7, including cover

Number of Tables in Supporting Information Section: 1

Number of Figures in Supporting Information Section: 5

Number of Tables: 1 (Tables S1)

Number of Figures: 5 (Figure S1 to Figure S5) 


\section{Contents}

Table S1. Information about the sampling locations and analytical results of ${ }^{239+240} \mathrm{Pu}$ activities, ${ }^{240} \mathrm{Pu} /{ }^{239} \mathrm{Pu}$ atom ratios, grain sizes and components of surface sediments.

Figure S1. The content of clay in surface sediments of river basins in southern China.

Figure S2. The schematic map of the North Equatorial Current-Kuroshio current system and the location of the Pacific Proving Ground (PPG) ${ }^{1-7}$.

Figure S3. Grain sizes, sediment components and ${ }^{239+240} \mathrm{Pu}$ activities in surface sediments of river basins in southern China.

Figure S4. Content of ${ }^{239+240} \mathrm{Pu}$ activity $(\mathrm{mBq} / \mathrm{g})$ and heavy metals $(\mathrm{mg} / \mathrm{kg})$ in surface sediments of river basins in southern China. The activities of ${ }^{239+240} \mathrm{Pu}$ in (a) are on a scale of 100 times, the activities of ${ }^{239+240} \mathrm{Pu}$ in (b) are the test results, and the activities of ${ }^{239+240} \mathrm{Pu}$ in (c) are on a scale of 1000 times.

Figure S5. Relationship between ${ }^{239+240} \mathrm{Pu}$ activity $(\mathrm{mBq} / \mathrm{g})$ and content of $\mathrm{As}(\mathrm{mg} / \mathrm{kg})$ found in surface sediments of river basins in southern China.

Table S1. Information about the sampling locations and analytical results of ${ }^{239+240} \mathrm{Pu}$ activities, ${ }^{240} \mathrm{Pu} /{ }^{239} \mathrm{Pu}$ atom ratios, grain sizes and components of surface sediments.

\begin{tabular}{|c|c|c|c|c|c|c|c|c|c|c|c|}
\hline Site & Longitude & Latitude & Region & $\begin{array}{l}\text { River } \\
\text { Basin }\end{array}$ & $\begin{array}{l}{ }^{239+240} \mathrm{Pu} \\
(\mathrm{mBq} / \mathrm{g})^{\mathrm{a}}\end{array}$ & $\begin{array}{l}{ }^{240} \mathrm{Pu} /{ }^{239} \mathrm{P} \\
\mathrm{u} \quad(\text { atom } \\
\text { ratio) }\end{array}$ & TOC & $\begin{array}{c}\text { Grain } \\
\text { size } \\
(\mu \mathrm{m})\end{array}$ & $\begin{array}{l}\text { Sand } \\
(\%)^{b}\end{array}$ & $\begin{array}{l}\text { silt } \\
(\%)^{b}\end{array}$ & $\begin{array}{l}\text { clay } \\
(\%)^{\mathrm{b}}\end{array}$ \\
\hline E02 & 113.273 & 23.998 & Beijiang & $\begin{array}{l}\text { Pearl } \\
\text { River }\end{array}$ & $\begin{array}{c}0.341 \pm \\
0.012\end{array}$ & $\begin{array}{c}0.199 \pm \\
0.032\end{array}$ & $3.81 \%$ & 2.53 & 87.27 & 10.95 & 1.78 \\
\hline E05 & 113.425 & 24.183 & Beijiang & $\begin{array}{l}\text { Pearl } \\
\text { River }\end{array}$ & $\begin{array}{c}0.305 \pm \\
0.021\end{array}$ & $\begin{array}{c}0.198 \pm \\
0.021\end{array}$ & $6.37 \%$ & 5.58 & 24.4 & 63.32 & 12.28 \\
\hline E08 & 114.269 & 23.161 & Dongjiang & $\begin{array}{l}\text { Pearl } \\
\text { River }\end{array}$ & $\begin{array}{c}0.283 \pm \\
0.035\end{array}$ & $\begin{array}{c}0.191 \pm \\
0.015\end{array}$ & $0.91 \%$ & - & - & - & - \\
\hline E11 & 115.259 & 24.154 & Dongjiang & $\begin{array}{l}\text { Pearl } \\
\text { River }\end{array}$ & $\begin{array}{c}0.336 \pm \\
0.015\end{array}$ & $\begin{array}{c}0.186 \pm \\
0.021\end{array}$ & $5.81 \%$ & 5.16 & 30.6 & 59.99 & 9.41 \\
\hline E13 & 115.779 & 23.963 & Hanjiang & $\begin{array}{l}\text { Pearl } \\
\text { River }\end{array}$ & $\begin{array}{c}0.391 \pm \\
0.035\end{array}$ & $\begin{array}{c}0.176 \pm \\
0.022\end{array}$ & $4.34 \%$ & 6.43 & 7.61 & 75.3 & 17.09 \\
\hline E14 & 116.101 & 24.241 & Hanjiang & $\begin{array}{l}\text { Pearl } \\
\text { River }\end{array}$ & $\begin{array}{c}0.321 \pm \\
0.042\end{array}$ & $\begin{array}{c}0.178 \pm \\
0.016\end{array}$ & $4.88 \%$ & 6.33 & 4.65 & 80.85 & 14.5 \\
\hline E15 & 116.141 & 24.646 & Hanjiang & $\begin{array}{l}\text { Pearl } \\
\text { River }\end{array}$ & $\begin{array}{c}0.259 \pm \\
0.053\end{array}$ & $\begin{array}{c}0.191 \pm \\
0.026\end{array}$ & $2.19 \%$ & 4.93 & 39.17 & 50.23 & 10.6 \\
\hline E26 & 116.653 & 23.683 & Hanjiang & $\begin{array}{l}\text { Pearl } \\
\text { River }\end{array}$ & $\begin{array}{c}0.690 \pm \\
0.067\end{array}$ & $\begin{array}{c}0.179 \pm \\
0.020\end{array}$ & $2.08 \%$ & 6.71 & 4.13 & 73.16 & 22.71 \\
\hline E27 & 116.464 & 23.461 & Hanjiang & $\begin{array}{l}\text { Pearl } \\
\text { River }\end{array}$ & $\begin{array}{c}1.792 \pm \\
0.134\end{array}$ & $\begin{array}{c}0.169 \pm \\
0.015\end{array}$ & $16.62 \%$ & 4.61 & 39.44 & 51.33 & 9.23 \\
\hline E28 & 115.619 & 22.955 & Hanjiang & $\begin{array}{l}\text { Pearl } \\
\text { River }\end{array}$ & $\begin{array}{c}0.483 \pm \\
0.243\end{array}$ & $\begin{array}{c}0.175 \pm \\
0.012\end{array}$ & $4.87 \%$ & 6.05 & 11.57 & 74.2 & 14.23 \\
\hline E30 & 114.462 & 23.04 & Dongjiang & $\begin{array}{l}\text { Pearl } \\
\text { River }\end{array}$ & $\begin{array}{c}0.788 \pm \\
0.059\end{array}$ & $\begin{array}{c}0.175 \pm \\
0.022\end{array}$ & $1.62 \%$ & 4.45 & 52.41 & 39.53 & 8.05 \\
\hline
\end{tabular}




\begin{tabular}{|c|c|c|c|c|c|c|c|c|c|c|c|}
\hline E31 & 113.846 & 23.104 & Dongjiang & $\begin{array}{l}\text { Pearl } \\
\text { River }\end{array}$ & $\begin{array}{c}3.322 \pm \\
0.499\end{array}$ & $\begin{array}{c}0.183 \pm \\
0.023\end{array}$ & $6.95 \%$ & 6.02 & 10.43 & 75.7 & 13.87 \\
\hline E35 & 112.999 & 22.503 & $\begin{array}{l}\text { Pearl } \\
\text { River }\end{array}$ & $\begin{array}{l}\text { Pearl } \\
\text { River }\end{array}$ & $\begin{array}{c}1.580 \pm \\
0.192\end{array}$ & $\begin{array}{c}0.172 \pm \\
0.031\end{array}$ & $7.23 \%$ & 5.47 & 24.03 & 65.03 & 10.94 \\
\hline E36 & 112.689 & 23.175 & $\begin{array}{l}\text { Pearl } \\
\text { River }\end{array}$ & $\begin{array}{l}\text { Pearl } \\
\text { River }\end{array}$ & $\begin{array}{c}0.043 \pm \\
0.013\end{array}$ & $\begin{array}{c}0.165 \pm \\
0.028\end{array}$ & $2.18 \%$ & - & - & - & - \\
\hline E38 & 112.808 & 23.189 & $\begin{array}{l}\text { Pearl } \\
\text { River }\end{array}$ & $\begin{array}{l}\text { Pearl } \\
\text { River }\end{array}$ & $\begin{array}{c}0.133 \pm \\
0.021\end{array}$ & $\begin{array}{c}0.184 \pm \\
0.026\end{array}$ & $5.93 \%$ & 6.19 & 10.36 & 72.87 & 16.77 \\
\hline E39 & 113.176 & 23.235 & $\begin{array}{l}\text { Pearl } \\
\text { River }\end{array}$ & $\begin{array}{l}\text { Pearl } \\
\text { River }\end{array}$ & $\begin{array}{c}0.011 \pm \\
0.019\end{array}$ & $\begin{array}{c}0.179 \pm \\
0.021\end{array}$ & $3.80 \%$ & 7.3 & 0.02 & 72.16 & 27.82 \\
\hline W47 & 110.555 & 21.927 & WGC & WGC & $\begin{array}{c}0.029 \pm \\
0.011\end{array}$ & $\begin{array}{c}0.182 \pm \\
0.016\end{array}$ & $3.80 \%$ & - & - & - & - \\
\hline W48 & 110.658 & 21.578 & WGC & WGC & $\begin{array}{c}0.056 \pm \\
0.028\end{array}$ & $\begin{array}{c}0.171 \pm \\
0.014\end{array}$ & $2.72 \%$ & 6.31 & 7.94 & 74.65 & 17.41 \\
\hline W54 & 110.369 & 20.642 & WGC & WGC & $\begin{array}{c}0.114 \pm \\
0.046\end{array}$ & $\begin{array}{c}0.215 \pm \\
0.021\end{array}$ & $3.38 \%$ & 5.82 & 12.41 & 75.5 & 12.09 \\
\hline W55 & 110.408 & 20.581 & WGC & WGC & $\begin{array}{c}0.339 \pm \\
0.022\end{array}$ & $\begin{array}{c}0.201 \pm \\
0.024\end{array}$ & $5.48 \%$ & 6.26 & 4.95 & 79.85 & 15.2 \\
\hline W58 & 110.783 & 21.455 & WGC & WGC & $\begin{array}{c}0.132 \pm \\
0.014\end{array}$ & $\begin{array}{c}0.187 \pm \\
0.021\end{array}$ & $4.51 \%$ & 5.8 & 13.74 & 73.73 & 12.53 \\
\hline W64 & 111.412 & 21.597 & WGC & WGC & $\begin{array}{c}0.094 \pm \\
0.014\end{array}$ & $\begin{array}{c}0.176 \pm \\
0.013\end{array}$ & $1.42 \%$ & 6.14 & 12.87 & 70.28 & 16.84 \\
\hline W69 & 111.819 & 21.953 & WGC & WGC & $\begin{array}{c}0.033 \pm \\
0.009\end{array}$ & $\begin{array}{c}0.169 \pm \\
0.014\end{array}$ & $2.45 \%$ & - & - & - & - \\
\hline W70 & 112.399 & 21.891 & WGC & WGC & $\begin{array}{c}0.066 \pm \\
0.024\end{array}$ & $\begin{array}{c}0.163 \pm \\
0.019\end{array}$ & $10.16 \%$ & 5.54 & 18.61 & 71.78 & 9.61 \\
\hline W72 & 112.015 & 21.83 & WGC & WGC & $\begin{array}{c}0.458 \pm \\
0.027\end{array}$ & $\begin{array}{c}0.212 \pm \\
0.012\end{array}$ & $9.53 \%$ & - & - & - & - \\
\hline W73 & 112.015 & 21.791 & WGC & WGC & $\begin{array}{c}4.676 \pm \\
0.075\end{array}$ & $\begin{array}{c}0.221 \pm \\
0.024\end{array}$ & $4.51 \%$ & 4.94 & 34.44 & 57.24 & 8.2 \\
\hline W74 & 111.914 & 21.686 & WGC & WGC & $\begin{array}{c}0.339 \pm \\
0.029\end{array}$ & $\begin{array}{c}0.215 \pm \\
0.021\end{array}$ & $7.43 \%$ & 5.25 & 30.2 & 59.42 & 10.38 \\
\hline E18 & 117.449 & 25.278 & Jinjiang & Jingjiang & $\begin{array}{c}0.396 \pm \\
0.020\end{array}$ & $\begin{array}{c}0.201 \pm \\
0.017\end{array}$ & $4.66 \%$ & 5.08 & 28.22 & 62.9 & 8.9 \\
\hline E19 & 118.33 & 25.3 & Jinjiang & Jingjiang & $\begin{array}{c}0.412 \pm \\
0.038\end{array}$ & $\begin{array}{c}0.192 \pm \\
0.026\end{array}$ & $2.20 \%$ & 5.06 & 33 & 58.9 & 8.1 \\
\hline E20 & 118.378 & 25.286 & Jinjiang & Jingjiang & $\begin{array}{c}0.403 \pm \\
0.065\end{array}$ & $\begin{array}{c}0.184 \pm \\
0.016\end{array}$ & $1.96 \%$ & 5.44 & 23.82 & 65.28 & 10.9 \\
\hline E21 & 118.591 & 24.877 & Jinjiang & Jingjiang & $\begin{array}{c}0.091 \pm \\
0.051\end{array}$ & $\begin{array}{c}0.168 \pm \\
0.027\end{array}$ & $4.42 \%$ & - & - & - & - \\
\hline E22 & 117.781 & 24.508 & Jinjiang & Jingjiang & $\begin{array}{c}0.561 \pm \\
0.036\end{array}$ & $\begin{array}{c}0.221 \pm \\
0.015\end{array}$ & $2.74 \%$ & 6.34 & 6.27 & 77.45 & 16.28 \\
\hline E23 & 117.755 & 24.488 & Jinjiang & Jingjiang & $\begin{array}{c}0.586 \pm \\
0.053\end{array}$ & $\begin{array}{c}0.207 \pm \\
0.013\end{array}$ & $1.42 \%$ & 5.43 & 23.58 & 65.64 & 10.78 \\
\hline E24 & 117.697 & 24.044 & Jinjiang & Jingjiang & $\begin{array}{c}0.218 \pm \\
0.028\end{array}$ & $\begin{array}{c}0.183 \pm \\
0.011\end{array}$ & $1.80 \%$ & 6.33 & 5.43 & 79.96 & 14.61 \\
\hline
\end{tabular}




\begin{tabular}{|c|c|c|c|c|c|c|c|c|c|c|c|}
\hline E25 & 117.334 & 23.973 & Jinjiang & Jingjiang & $\begin{array}{c}0.430 \pm \\
0.128\end{array}$ & $\begin{array}{c}0.167 \pm \\
0.014\end{array}$ & $3.30 \%$ & 7.05 & 1.07 & 75.4 & 23.53 \\
\hline E40 & 119.123 & 26.145 & Minjiang & Minjiang & $\begin{array}{c}0.289 \pm \\
0.055\end{array}$ & $\begin{array}{c}0.222 \pm \\
0.011\end{array}$ & $0.73 \%$ & 6.11 & 10.19 & 74.44 & 15.37 \\
\hline W08 & 111.504 & 23.383 & Hongshui & $\begin{array}{l}\text { Pearl } \\
\text { River }\end{array}$ & $\begin{array}{c}0.012 \pm \\
0.017\end{array}$ & $\begin{array}{c}0.171 \pm \\
0.026\end{array}$ & $2.72 \%$ & 6.63 & 2.29 & 79.6 & 18.11 \\
\hline W11 & 110.532 & 24.775 & Hongshui & $\begin{array}{l}\text { Pearl } \\
\text { River }\end{array}$ & $\begin{array}{c}0.014 \pm \\
0.034\end{array}$ & $\begin{array}{c}0.169 \pm \\
0.024\end{array}$ & $2.06 \%$ & 6.6 & 4.15 & 76.97 & 18.88 \\
\hline W12 & 110.534 & 24.784 & Hongshui & $\begin{array}{l}\text { Pearl } \\
\text { River }\end{array}$ & $\begin{array}{c}0.065 \pm \\
0.020\end{array}$ & $\begin{array}{c}0.221 \pm \\
0.021\end{array}$ & $5.32 \%$ & 7.84 & 0 & 57.05 & 42.95 \\
\hline W15 & 108.559 & 24.531 & Hongshui & $\begin{array}{l}\text { Pearl } \\
\text { River }\end{array}$ & $\begin{array}{c}0.083 \pm \\
0.042\end{array}$ & $\begin{array}{c}0.174 \pm \\
0.018\end{array}$ & $1.59 \%$ & - & - & - & - \\
\hline W16 & 108.554 & 24.531 & Hongshui & $\begin{array}{l}\text { Pearl } \\
\text { River }\end{array}$ & $\begin{array}{c}0.117 \pm \\
0.022\end{array}$ & $\begin{array}{c}0.168 \pm \\
0.016\end{array}$ & $4.54 \%$ & 6.32 & 6.92 & 77.2 & 15.89 \\
\hline W17 & 107.989 & 23.732 & Hongshui & $\begin{array}{l}\text { Pearl } \\
\text { River }\end{array}$ & $\begin{array}{c}0.109 \pm \\
0.024\end{array}$ & $\begin{array}{c}0.196 \pm \\
0.014\end{array}$ & $4.29 \%$ & 6.01 & 12.02 & 72.13 & 15.85 \\
\hline W20 & 105.803 & 24.705 & Hongshui & $\begin{array}{l}\text { Pearl } \\
\text { River }\end{array}$ & $\begin{array}{c}0.127 \pm \\
0.018\end{array}$ & $\begin{array}{c}0.172 \pm \\
0.024\end{array}$ & $6.91 \%$ & 5.67 & 16.52 & 72.07 & 11.41 \\
\hline W22 & 105.787 & 25.381 & Hongshui & $\begin{array}{l}\text { Pearl } \\
\text { River }\end{array}$ & $\begin{array}{c}0.253 \pm \\
0.104\end{array}$ & $\begin{array}{c}0.189 \pm \\
0.012\end{array}$ & $5.30 \%$ & 7.28 & 0 & 70.14 & 29.86 \\
\hline W23 & 105.483 & 25.206 & Hongshui & $\begin{array}{l}\text { Pearl } \\
\text { River }\end{array}$ & $\begin{array}{c}0.269 \pm \\
0.051\end{array}$ & $\begin{array}{c}0.190 \pm \\
0.021\end{array}$ & $8.41 \%$ & 5.54 & 2.67 & 78.79 & 18.54 \\
\hline W24 & 106.208 & 24.316 & Hongshui & $\begin{array}{l}\text { Pearl } \\
\text { River }\end{array}$ & $\begin{array}{c}0.122 \pm \\
0.046\end{array}$ & $\begin{array}{c}0.179 \pm \\
0.014\end{array}$ & $2.73 \%$ & 5.76 & 18.97 & 67.7 & 13.33 \\
\hline W25 & 106.647 & 23.871 & Hongshui & $\begin{array}{l}\text { Pearl } \\
\text { River }\end{array}$ & $\begin{array}{c}0.114 \pm \\
0.040\end{array}$ & $\begin{array}{c}0.199 \pm \\
0.021\end{array}$ & $4.79 \%$ & - & - & - & - \\
\hline W26 & 107.351 & 22.412 & Hongshui & $\begin{array}{l}\text { Pearl } \\
\text { River }\end{array}$ & $\begin{array}{c}0.093 \pm \\
0.012\end{array}$ & $\begin{array}{c}0.175 \pm \\
0.032\end{array}$ & $1.51 \%$ & 2.81 & 90.66 & 7.04 & 2.31 \\
\hline W29 & 108.187 & 22.82 & Hongshui & $\begin{array}{l}\text { Pearl } \\
\text { River }\end{array}$ & $\begin{array}{c}0.190 \pm \\
0.047\end{array}$ & $\begin{array}{c}0.212 \pm \\
0.022\end{array}$ & $4.15 \%$ & 6.26 & 8.73 & 74.9 & 16.37 \\
\hline W34 & 110.568 & 22.861 & Hongshui & $\begin{array}{l}\text { Pearl } \\
\text { River }\end{array}$ & $\begin{array}{c}0.079 \pm \\
0.008\end{array}$ & $\begin{array}{c}0.181 \pm \\
0.021\end{array}$ & $7.53 \%$ & - & - & - & - \\
\hline HN08 & 110.816 & 19.585 & & $\begin{array}{c}\text { Hainan } \\
\text { rivers }\end{array}$ & $\begin{array}{c}0.076 \pm \\
0.015\end{array}$ & $\begin{array}{c}0.168 \pm \\
0.025\end{array}$ & $0.65 \%$ & - & - & - & - \\
\hline HN09 & 110.592 & 19.154 & & $\begin{array}{c}\text { Hainan } \\
\text { rivers }\end{array}$ & $\begin{array}{c}0.034 \pm \\
0.011\end{array}$ & $\begin{array}{c}0.172 \pm \\
0.022\end{array}$ & $1.14 \%$ & - & - & - & - \\
\hline HN12 & 110.035 & 18.522 & & $\begin{array}{c}\text { Hainan } \\
\text { rivers }\end{array}$ & $\begin{array}{c}0.057 \pm \\
0.021\end{array}$ & $\begin{array}{c}0.167 \pm \\
0.016\end{array}$ & $0.53 \%$ & - & - & - & - \\
\hline
\end{tabular}

${ }^{\mathrm{a}}$ All given uncertainties are \pm two standard deviation errors.

${ }^{\mathrm{b}}$ Diameter ranges: clay particles, $0.02 \mu \mathrm{m}$ to $64 \mu \mathrm{m}$; silt particles, $64 \mu \mathrm{m}$ to $128 \mu \mathrm{m}$; sand particles, $128 \mu \mathrm{m}$ to $2000 \mu \mathrm{m}$. 

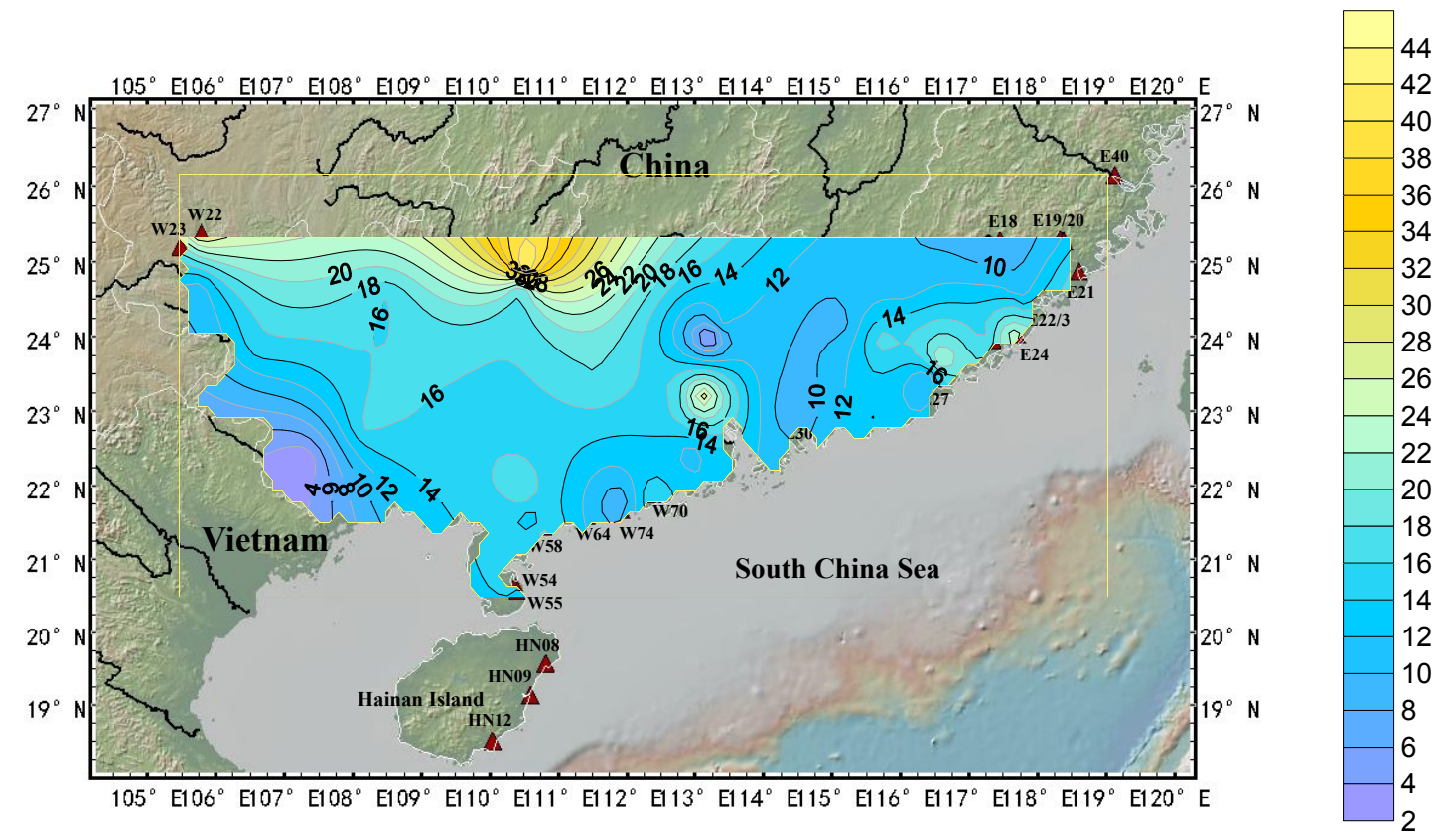

Figure S1. The content of clay in surface sediments of river basins in southern China.

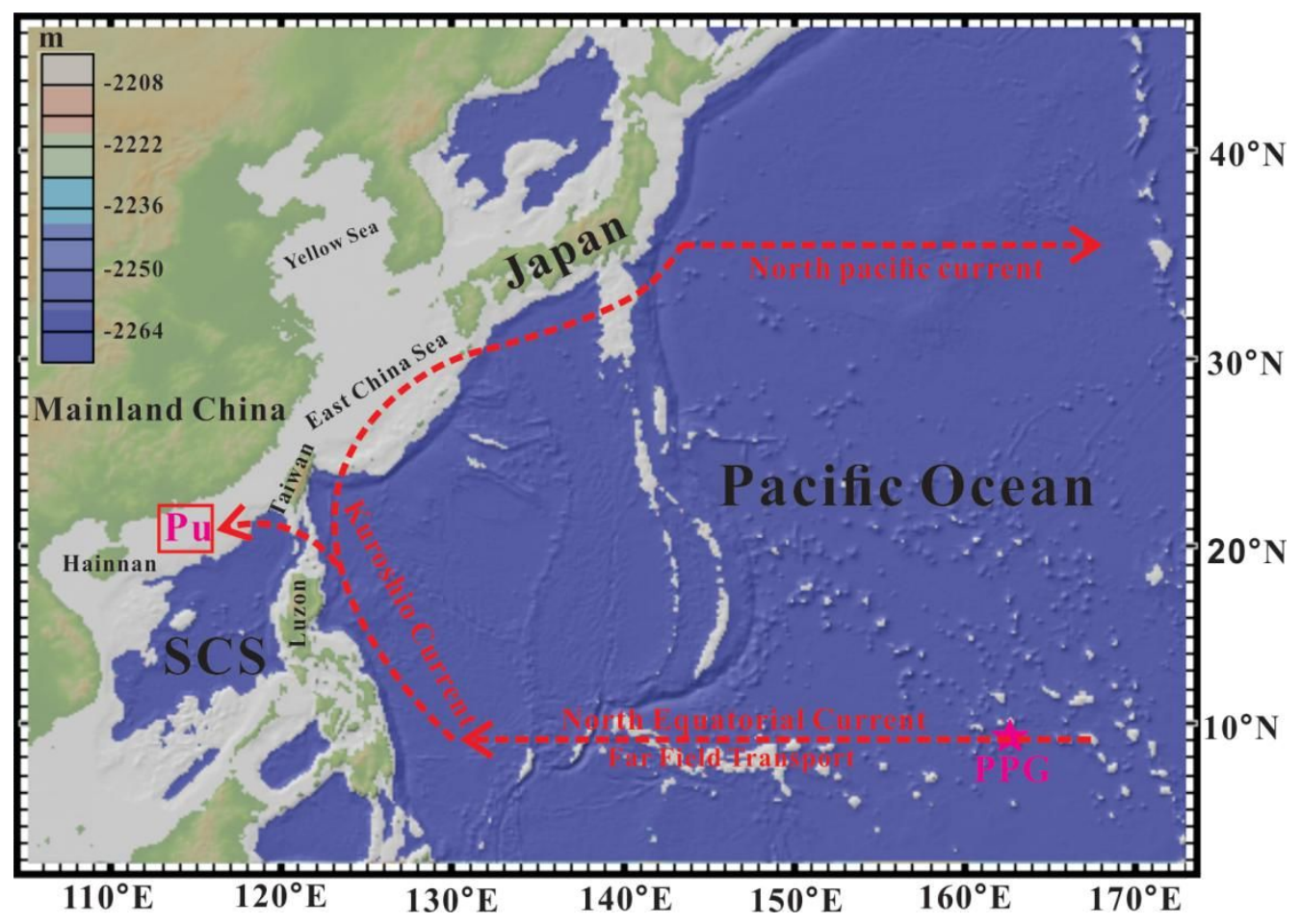

Figure S2. The schematic map of the North Equatorial Current-Kuroshio current system and the location of the Pacific Proving Ground (PPG). ${ }^{1-2}$ 

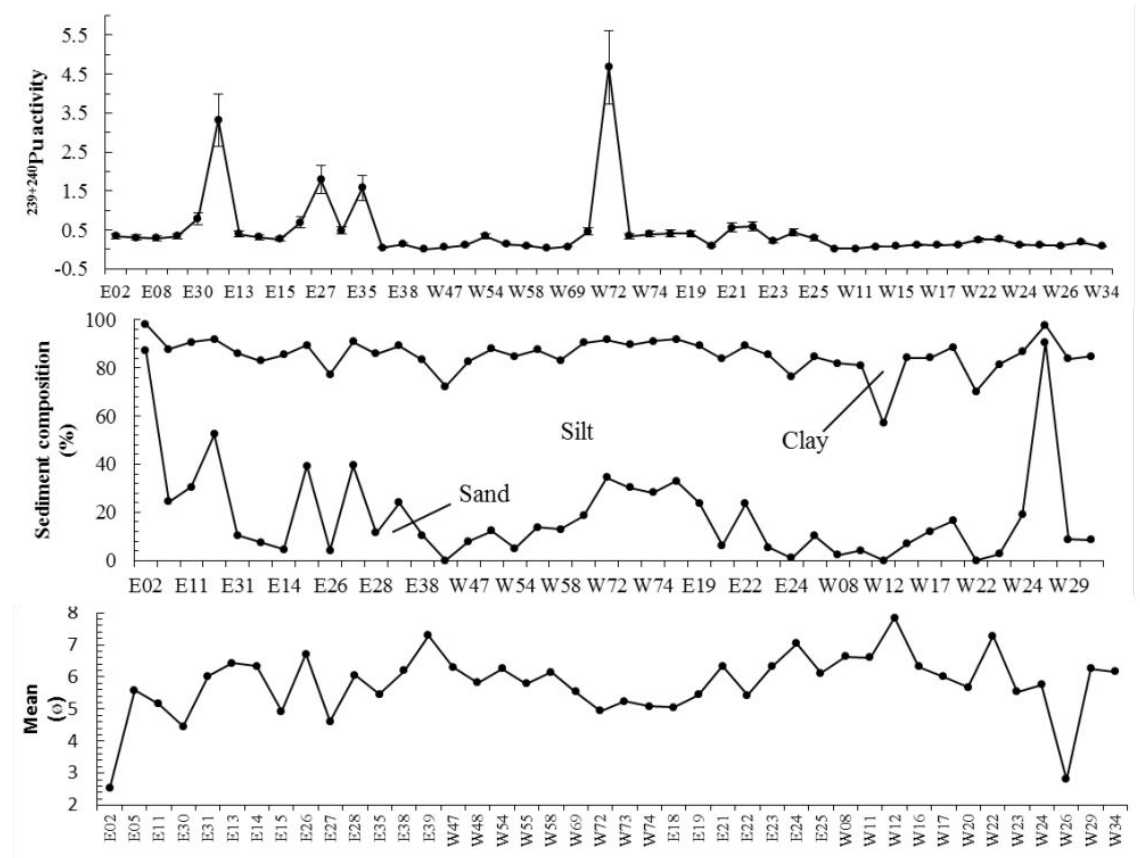

Figure S3. Grain sizes, sediment components and ${ }^{239+240} \mathrm{Pu}$ activities in surface sediments of river basins in southern China.
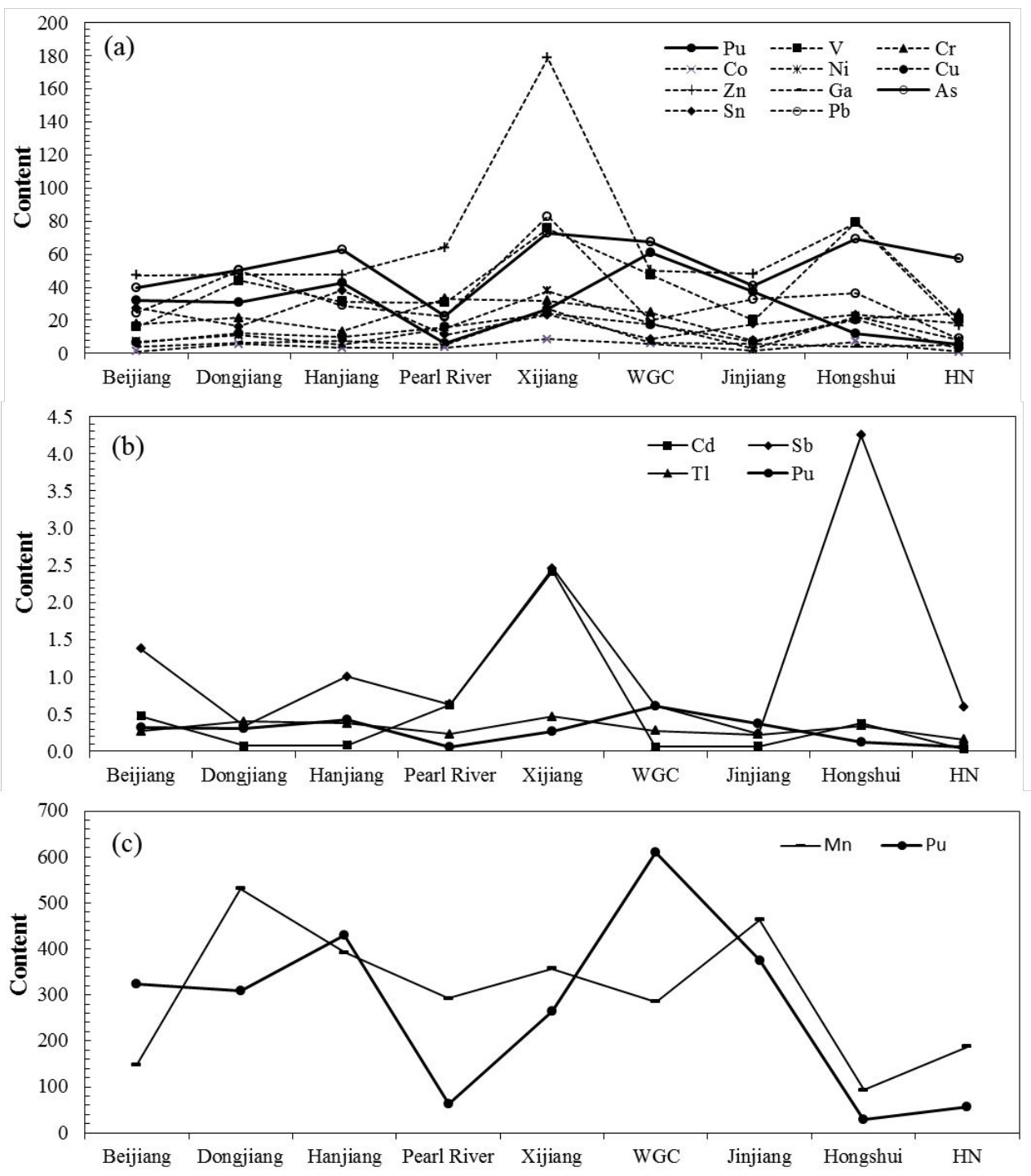
Figure S4. Content of ${ }^{239+240} \mathrm{Pu}$ activity $(\mathrm{mBq} / \mathrm{g})$ and heavy metals $(\mathrm{mg} / \mathrm{kg})$ in surface sediments of river basins in southern China. The activities of ${ }^{239+240} \mathrm{Pu}$ in (a) are on a scale of 100 times, the activities of ${ }^{239+240} \mathrm{Pu}$ in (b) are the test results, and the activities of ${ }^{239+240} \mathrm{Pu}$ in (c) are on a scale of 1000 times.

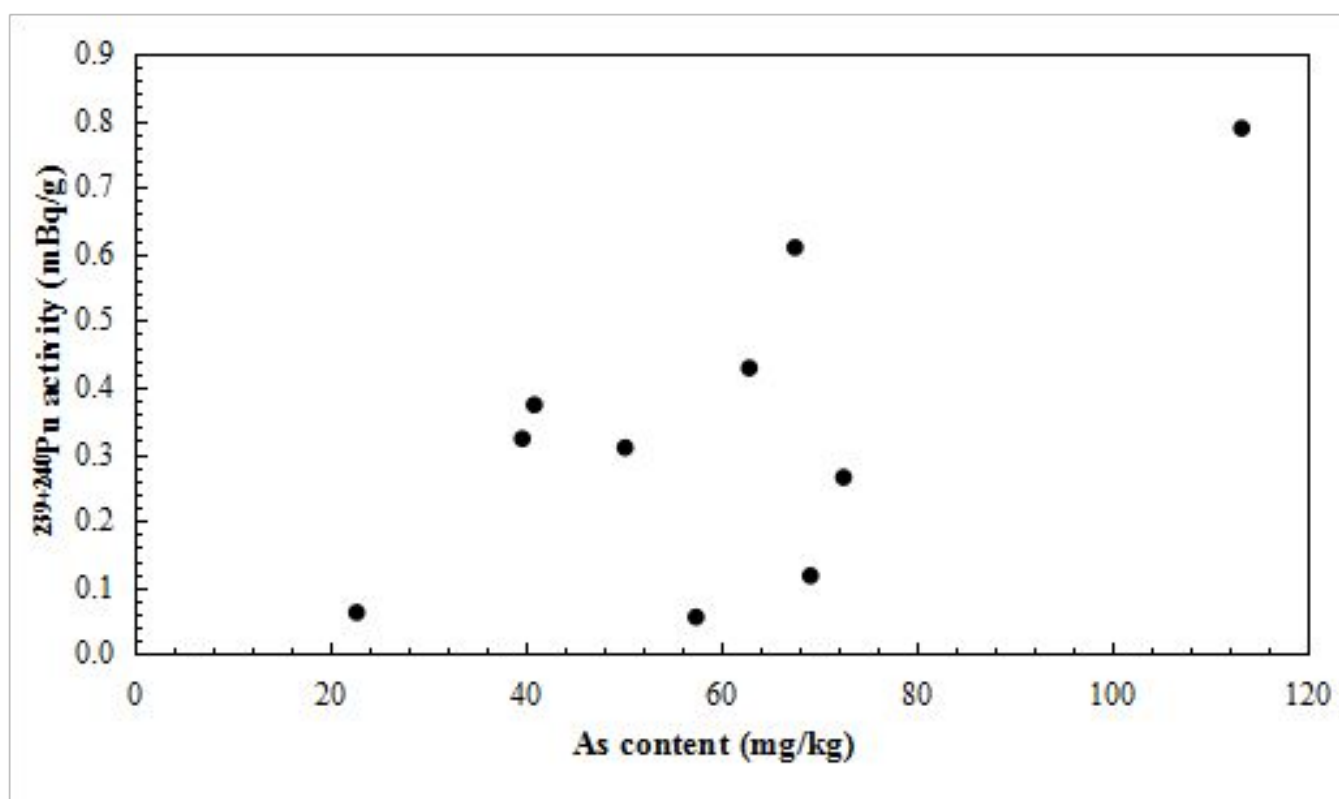

Figure S5. Relationship between ${ }^{239+240} \mathrm{Pu}$ activity $(\mathrm{mBq} / \mathrm{g})$ and content of $\mathrm{As}(\mathrm{mg} / \mathrm{kg})$ found in surface sediments of river basins in southern China.

\section{References}

(1) Wu, J.; Zheng, J.; Dai, M.; Huh, C. A.; Chen, W.; Tagami, K.; Uchida, S. Isotopic composition and distribution of plutonium in northern South China Sea sediments revealed continuous release and transport of Pu from the Marshall Islands. Environ. Sci. Technol. 2014, 48 (6), 3136-3144. DOI: 10.1021/es405363q.

(2) Wu, J.; Dai, M.; Xu, Y.; Zheng, J. Sources and accumulation of plutonium in a large Western Pacific marginal sea: The South China Sea. Sci. Total. Environ. 2018, 610, 200-211. 